\title{
Distribution of a water-soluble porphyrin in mixed monolayers evaluated by energy transfer
}

\author{
María Teresa Martín* and Dietmar Möbius \\ Max-Planck-Institut für biophysikalische Chemie, Am Faberg 11, D-37077 Göttingen, \\ Germany \\ (Received 6 September 1996; revised 5 January 1997)
}

\begin{abstract}
Mixed monolayers of a water-soluble tetra-anionic porphyrin (PO) with dioctadecyldimethylammonium bromide (DOMA) and methyl stearate (SME) have been formed by co-spreading, with the aim of achieving molecular recognition. The lateral distribution of PO in such monolayers has been investigated by Förster energy transfer from the amphiphilic oxacyanine dye $S 9$ in a mixed monolayer of methyl arachidate (AME) and arachidic acid ( $A \Lambda$ ). Mixed monolaycrs of PO:DOMA:SME, mixing ratios 1:4:X $(X=32,64,160,240,320$ and 480) were transferred to hydrophobic glass plates coated with $\mathrm{S} 9: \mathrm{AME}: \mathrm{AA}=1: 2: 18$ by vertical dipping. The system was excited with ultraviolet radiation $(\lambda=366 \mathrm{~nm})$. The excitation energy of $S 9$ is transferred to PO and the relative fluorescence intensity $I_{d} / I_{\infty}\left(I_{d}\right.$ in the presence and $I_{\infty}$ in the absence of PO) of S9 at $420 \mathrm{~nm}$ was measured. The results indicate a homogeneous lateral distribution of PO, i.e. no cluster formation, for low densities of PO in mixed monolayers with DOMA and SME. (C) 1997 Elsevier Science Ltd. All rights reserved.
\end{abstract}

(Keywords: energy transfer; molecular distribution; porphyrin)

\section{INTRODUCTION}

Mixed monolayers with functionalized molecules play an important role as model systems for the investigation and control of complex reactions and processes at the molecular level. In biological systems the molecules are in a specific order, and entirely new properties are caused by this organisation at molecular level. The Langmuir-Blodgett (LB) method offers a way to construct organised arrangements of molecules acting as molecular functional units ${ }^{1}$ that have useful properties depending on the details of the geometrical arrangement and the interactions between the molecular components.

One of the most important pieces of structural information is the lateral distribution of the functionalised molecules in a mixed monolayer. Details of the arrangement of molecules in systems of monolayers have been evaluated by using Förstertype energy transfer from an excited donor to an acceptor $^{2,3}$ in studies of deactivation processes, sensitisation processes, rearrangement processes in monolayer systems, manipulation of monolayers,

* To whom correspondence should be addressed.

On leave of absence from the Departamento de Quimica Física Termodinámica Aplicada, Facultad de Ciencias, Avda. San Alberto Magno s/n, 14004 Córdoba, Spain diffusion processes in layer systems, determination of distances in biological systems ${ }^{4}$ and lateral distribution of dyes in monolayers and clustcr formation ${ }^{5}$. Most of these studies have been accomplished with amphiphilic cyanines dyes both as donor and acceptor.

In this work, we present the analysis by Förster-type energy transfer of the distribution of porphyrin molecules in mixed monolayers. A water-soluble tetraanionic porphyrin (PO) has been optimally bound to the amphiphilic matrix of dioctadecyldimethylammonium bromide (DOMA) as anchor and methyl stearate (SME) as neutral component, by lateral organization of the anchor to match the charge geometry of the PO. Enhanced binding of PO by such laterally organized monolayers, compared with binding by a non-organized matrix of the same composition, has been observed ${ }^{6}$. The mixed monolayers of PO:DOMA:SME, of different porphyrin surface densities, were deposited by LB transfer on a solid substrate coated with a donor layer (S9:AME:AA = 1:2:18). The system was excited with ultraviolet (UV) radiation and the excitation energy was transferred from S9 to PO, thus quenching the fluorescence of the donor S9. The dependence of the quenching on the surface density of PO molecules provides information on the lateral distribution of PO. 


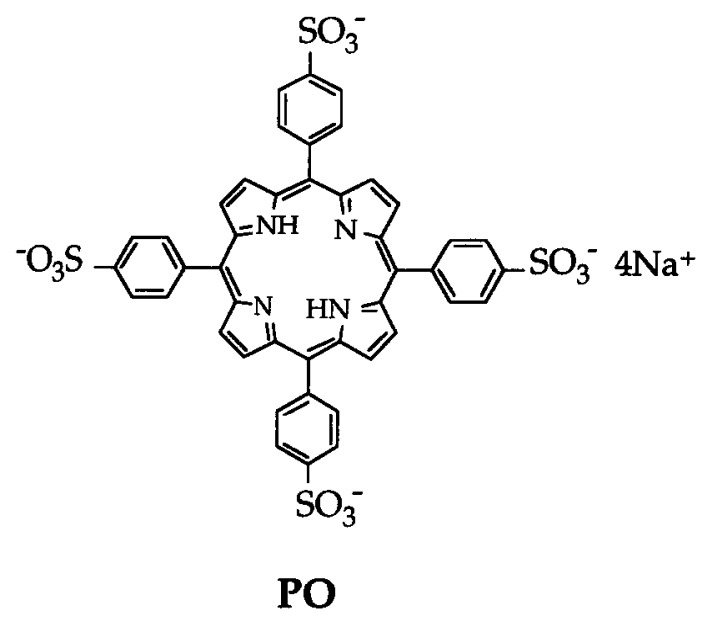

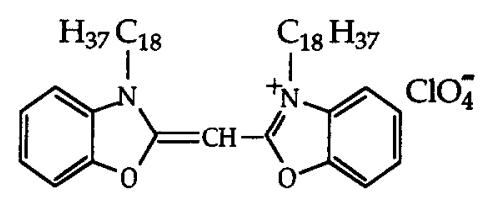

S9

Scheme 1

\section{EXPERIMENTAL}

Methyl stearate (SME) and methyl arachidate (AME) were purchased from E. Merck and used as received. Arachidic acid (AA) was purchased from E. Merck and recrystallized from ethanol. Dioctadecyldimethylammonium bromide (DOMA) was used as received (Sigma Chemical Co.). Tetrasodiummeso-tetra(4-sulfonatophenyl) porphine (PO) was obtained from Strem and used without further purification (structure in Scheme 1). Eicosylamine (EA) was prepared by W. Schulten in our laboratory. The cyanine dye S9 was synthesized by $\mathbf{J}$. Sondermann ${ }^{7}$ (see Scheme l). Hexamethyldisilazane was purchased from Fluka and used as received to silanize the glass plates. Chloroform and a mixture of dichloromethane:methanol: $\mathrm{H}_{2} \mathrm{O}$, volume ratio 15:9:2, were used as spreading solvents and werc obtained from Baker Chemicals. The water used for the subphase was obtained from a Milli-Q filtration unit (Millipore Corp.).

Monolayers of PO:DOMA:SME, molar ratio 1:4:X $(X=32,64,160,240,320$ and 480$)$, were prepared by co-spreading in circular troughs ${ }^{8}$. The temperature of the subphase was kept at $21^{\circ} \mathrm{C}$. The monolayers were compressed by a movable Teflon barrier with a com pression velocity of $0.04-$ $0.06 \mathrm{~nm}^{2} \min ^{-1}$ molecule ${ }^{-1}$ and transferred on silanized glass plates at a surface pressure of $30 \mathrm{mN} \mathrm{m}^{-1}$ by using the technique of vertical dipping. The transferred systems were characterized by absorption spectroscopy and used for energy transfer measurements. Two types of system were assembled by sequential monolayer transfer ( $\downarrow$ immersion, $\uparrow$ withdrawal). (a) For the

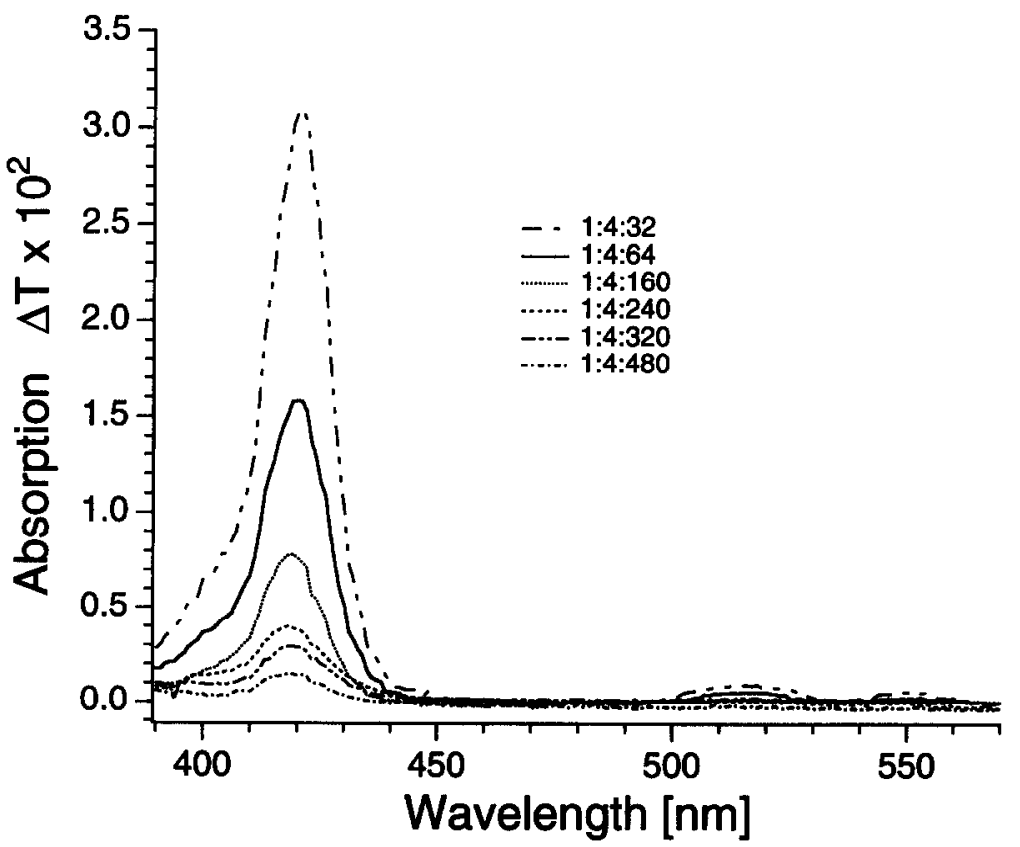

Figure 1 Absorption spectra of mixed monolayers of PO:DOMA:SME, molar ratios as indicated in the plot, transferred on silanized glass plates at $30 \mathrm{mN} \mathrm{m}^{-1} ; T=21^{\circ} \mathrm{C}$ 
determination of critical distance: $\downarrow$ EA, $\uparrow$ S9:AME:AA in the ratio 1:2:18, $\downarrow$ PO:DOMA:SME $=1: 4: X$ (AA in the reference section) and $\uparrow \mathrm{AME}$; (b) for evaluation of the porphyrin distribution: $\downarrow$ EA, $\uparrow$ AA, $\downarrow$ S9:AME:AA $=1: 2: 18$ and $\uparrow$ PO:DOMA:SME $=1: 4: X$ (AME: $\mathrm{AA}=1: 9$ in the reference section).

Details of the apparatus for measuring the spectral distribution and intensity of fluorescence of monolayer assemblies have been given elsewhere'

\section{RESULTS AND DISCUSSION}

The negatively charged PO molecules, after cospreading with a mixture of DOMA (positively charged) and SME (neutral), are optimally bound as has been described in detail elsewhere ${ }^{6}$. Mixed monolayers of PO:DOMA:SME of different porphyrin surface density were transferred on silanized glass with a transfer ratio of $\approx 1$. The absorption spectra, measured as the difference $\Delta T$ between a reference section without the PO monolayer and the sample section, for normal incidence of the transferred monolayers are shown in Figure 1. The shapc and maximum wavelength of the Soret band, $\lambda_{\max } \approx 418 \mathrm{~nm}$, of all spectra are in agreement with those described previously ${ }^{6}$. Accordingly, the PO molecules are in the monomeric form in the different mixtures, but absorption spectroscopy does not provide information on the lateral distribution of the PO molecules in the mixed monolayer; i.e. a homogeneous distribution and cluster formation are both possible.

To discriminate between these alternatives, we studied Förster energy transfer from the cyanine $S 9$ as donor, in a mixed monolayer S9:AME:AA = 1:2:18, to $P O$ as acceptor, in mixed monolayers PO:DOMA:SME = 1:4: $X \quad(X=32,64,160,240,320$ and 480 ). The system was excited with UV radiation, $\max =366 \mathrm{~nm}$. The excitation energy of $\mathrm{S} 9$ is transferred to $P O$ and the relative fluorescence intensity $I_{d} / I_{\infty}$ ( $I_{d}$ in the presence and $I_{\infty}$ in the absence of PO) of S9 at $420 \mathrm{~nm}$ was measured.

The fluorescence intensity, $I_{d}$, for a distance $d$ between the planes of chromophores of the donor and the acceptor respectively, is given by ${ }^{10}$ :

$$
\begin{aligned}
& \left(\frac{I_{d}}{I_{\infty}}\right)_{\mathrm{D}}=\left[1+\left(\frac{d_{0}}{d}\right)^{4}\right]^{-1} \\
& d_{0}=\alpha\left(\frac{\lambda_{\mathrm{D}}}{n}\right)\left(q_{\mathrm{D}} \mathrm{A}\right)^{1 / 4}
\end{aligned}
$$

where $d_{0}$ is the critical distance in monolayer systems; $\alpha$ is a numerical factor depending on the relative orientation of the transition moments of donor (D) and acceptor (A) (here, $\alpha=0.098)^{4} ; q_{\mathrm{D}}$ is the probability of fluorescence emission of the donor in the emitting state in the absence of the acceptor
$\left(q_{\mathrm{D}}=0.6\right)^{4} ; \lambda_{\mathrm{D}}$ is the wavelength of fluorescence of the donor $\left(\lambda_{\mathrm{D}}=420 \mathrm{~nm}\right) ; n$ is the refractive index of the medium $(n=1.5) ; A_{\mathrm{A}}$ is the absorption of the acceptor at $\lambda_{\mathrm{D}}\left(A_{\mathrm{A}} \approx \Delta T\right)$. These equations are based on the assumption of a homogeneous distribution of the acceptor molecules in the layer, e.g. high acceptor surface densities, $\sigma_{\mathrm{A}}$, when energy transfer from an excited donor molecule can take place to many acceptor molecules with comparable rates. However, this assumption is not justified for very small values of $\sigma_{\mathrm{A}}$ (ref. 4). At small surface acceptor densities, only the energy transfer from the donor molecule to the nearest acceptor molecule (distance $R$ ) needs to be considered. The relative fluorescence intensity of S9 for $\sigma_{\mathrm{A}}$ in the layer is given by Equations (3)-(5) ${ }^{9}$ :

$$
\begin{aligned}
& P=\left[1+\left(\frac{R}{R_{0}}\right)^{6}\right]^{-1} \\
& R_{0}^{6}=F \cdot \frac{d_{0}^{4}}{\sigma_{\mathrm{A}}}
\end{aligned}
$$

where $P$ is defined as the probability of energy transfer (Förster); $R_{0}$ is the critical Förster radius; $\sigma_{\mathrm{A}}$ is the surface density of acceptor; and $F$ is the numerical factor depending on the orientation of the transition moments of donor and acceptor. The chromophores of the cyanine are statistically oriented in the layer plane'. The porphyrin ring lies flat according to absorption spectra (not shown here) measured with plane-polarized light under both $45^{\circ}$ and $30^{\circ}$ incidence. In this case, $F=2.12$ (ref. 4). For a small distance between the layers of donor and acceptor, $d \approx 0$, and $R \approx 1 / 2\left(\sqrt{\sigma_{\mathrm{A}}}\right)^{-1}$ the relative fluorescence intensity of the donor is:

$$
\left(\frac{I_{d \approx 0}}{I_{\infty}}\right)_{\mathrm{D}}=1-P=1-\left[1+\left(\frac{1}{2 R_{0} \cdot \sqrt{\sigma_{\Lambda}}}\right)^{6}\right]^{-1}
$$

When the critical distance in monolayer assemblies, $d_{0}$, has been determined, the theoretical dependence of the relative fluorescence intensity $\left(I_{d \approx 0} / I_{\infty}\right)_{\mathrm{D}}$ of the donor according to equation (5) can be compared with experimental data in order to characterize the acceptor distribution for small $\sigma_{\mathrm{A}}$ in monolayer assemblies.

In monolayer assemblies (a) (described in Experimental), the values of $\left(I_{5.4} / I_{\infty}\right)_{\mathrm{s} 9}$ were measured for different porphyrin surface densities (PO:DOMA:SME = 1:4: $X, X=32,64$ and 160). The $d_{0}$ values were calculated from equation (1) and the Förster radius, $\boldsymbol{R}_{0}$, was determined from equation (4). These results are shown in Table 1 , and the average value is $R_{0}=5.73 \mathrm{~nm}$.

In this type of measurements, the absorption of the porphyrin monolayer at $366 \mathrm{~nm}$ (excitation wavelength of the cyanine dye) and $420 \mathrm{~nm}$ (fluorescence maxima of S9), in view of the trivial reabsorption by the porphyrin (maximum $\Delta T \approx 0.03$, see Figure 1 ), has no 


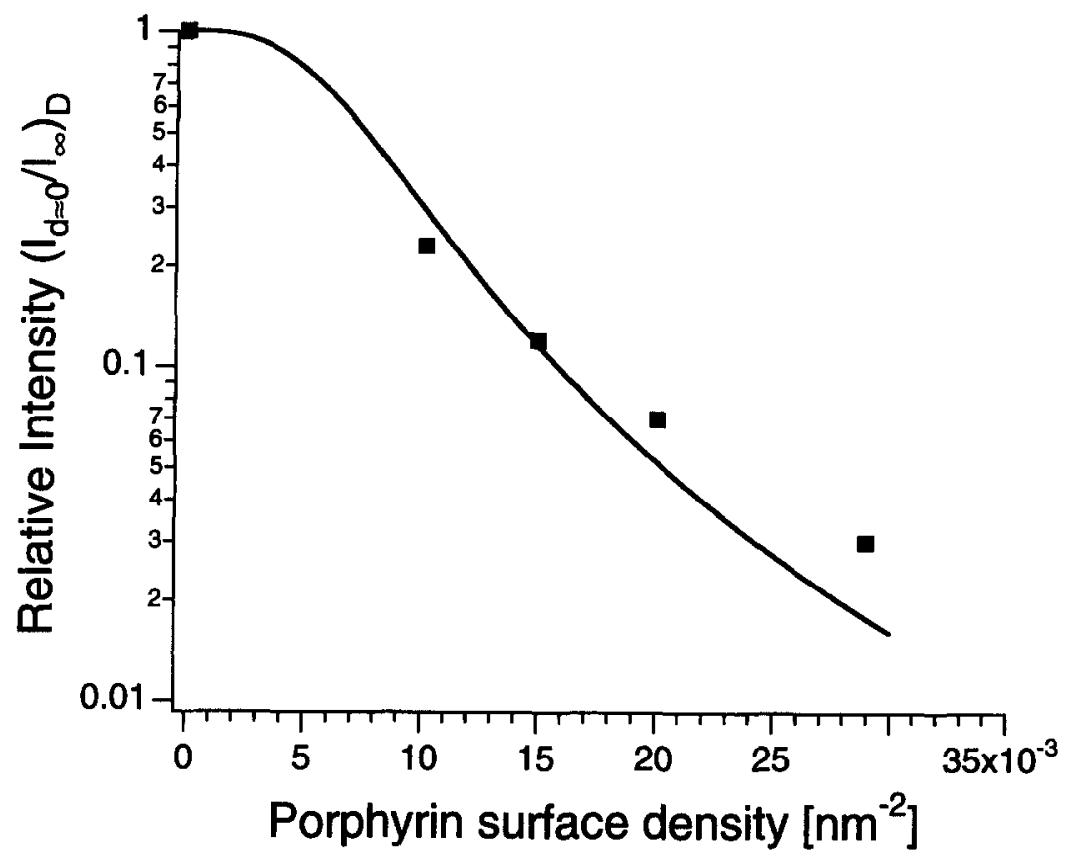

Figure 2 Comparison of the experimental results, $\left(I_{d \approx 0} / I_{\infty}\right)_{\mathrm{S} 9}$ (full squares), with theoretical curve [from equation (5) with $R_{0}=5.73 \mathrm{~nm}$, solid line]. The data are plotted against the porphyrin surface density in PO:DOMA:SME mixed monolayers

Table 1 Determination of critical parameters

\begin{tabular}{lcllll}
\hline Acceptor $^{a}$ & $\begin{array}{l}\text { Area }^{b} \\
\left(\mathrm{~nm}^{2}\right)\end{array}$ & $\begin{array}{l}\sigma_{\mathrm{A}} \\
\left(\mathrm{nm}^{-2}\right)\end{array}$ & $\left(I_{5.4} / I_{\infty}\right)_{\mathrm{sg}}$ & $\begin{array}{l}d_{0}{ }^{c} \\
(\mathrm{~nm})\end{array}$ & $\begin{array}{l}R_{0}{ }^{d} \\
(\mathrm{~nm})\end{array}$ \\
\hline $1: 4: 32$ & 8.0 & 0.125 & 0.26 & 7.01 & 5.86 \\
$1: 4: 64$ & 14.4 & 0.069 & 0.39 & 6.04 & 5.84 \\
$1: 4: 160$ & 33.6 & 0.029 & 0.69 & 4.42 & 5.49 \\
\hline
\end{tabular}

${ }^{a}$ Mixed monolayers ratios: PO:DOMA:SME

${ }^{5}$ Area per PO molecule calculated with $0.2 \mathrm{~nm}^{2}$ (SME molecule) ${ }^{-1}$ and $0.4 \mathrm{~nm}^{2}$ (DOMA molecule) ${ }^{-1}$

${ }^{c}$ Calculated values from equation ( 1 )

${ }^{d}$ Calculated value from equation (4)

Donor: S9:AME:AA = 1:2:18

Table 2 Evaluation of porphyrin distribution

\begin{tabular}{llll}
\hline Acceptor & $\sigma_{\mathrm{A}}\left(\mathrm{nm}^{-2}\right)$ & $\begin{array}{l}\left(I_{d \approx 0} / I_{\infty}\right)_{\mathrm{sg}}, \\
\text { calculated }^{a}\end{array}$ & $\begin{array}{l}\left(I_{d \approx 0} / I_{\infty}\right)_{\mathrm{s},}, \\
\text { experimental }\end{array}$ \\
\hline $1: 4: 32$ & 0.125 & $2.00 \times 10^{-4}$ & $\approx 0$ \\
$1: 4: 64$ & 0.069 & $1.20 \times 10^{-3}$ & $\approx 0$ \\
$1: 4: 160$ & 0.029 & $\mathbf{0 . 0 2}$ & $\mathbf{0 . 0 3}$ \\
$1: 4: 240$ & 0.020 & $\mathbf{0 . 0 5}$ & $\mathbf{0 . 0 7}$ \\
$:: 4: 320$ & 0.015 & $\mathbf{0 . 1 1 ^ { h }}$ & $\mathbf{0 . 1 2}$ \\
$1: 4: 480$ & 0.010 & $\mathbf{0 . 2 9}$ & $\mathbf{0 . 2 3}$ \\
\hline
\end{tabular}

"With equation (5)

${ }^{b}$ Calculated with $R_{0}=5.73 \mathrm{~nm}$ (average $R_{0}$ )

Donor: S9:AME:AA = 1:2:18

detectable influence in the determination of the relative fluorescence intensities of S9.

Then, the relative donor fluorescence intensities $\left(I_{d \approx 0} / I_{\infty}\right)_{\mathrm{S} 9}$ in systems (b) (described in Experimental) were measured. The experimental values of $\left(I_{d \approx 0} / I_{\infty}\right)_{\mathrm{S} 9}$ are compared in Table 2 with the values calculated from equation (5).
The theoretical curve (with $R_{0}=5.73 \mathrm{~nm}$, solid line) and the experimental values (full squares) are plotted versus porphyrin surface density in Figure 2. The agreement between theory and experiment is a direct demonstration for the homogeneous distribution of the PO molecules in monolayers formed by co-spreading with the amphiphilic matrix of DOMA and SME.

\section{ACKNOWLEDGEMENTS}

Support of this work by a fellowship of the Ministerio de Educación y Ciencia of Spain for one of us (Dr María T. Martín) is gratefully acknowledged. We wish to thank Mrs G. Debuch for technical assistance.

\section{REFERENCES}

1. Kuhn, H., Pure Appl. Chem., 1965, 11, 345.

2. Förster, T., in Fluoreszenz Organischer Verbindungen, 1st edn. Vandenhoeck and Ruprecht, Göttingen, 1951.

3. Förster, T., Naturwiss., 1945, 33, 166.

4. Kuhn, H. and Möbius, D., in Investigations of Surfaces and Interfaces, Vol. IXB, 2nd edn, ed. B. W. Rossiter and R. C. Baetzold. John Wiley and Sons, Inc., New York, 1993, p. 375 .

5. Bücher, H. V., Elsner, O., Möbius, D., Tillmann, P. and Wiegand, J. Z., Physik. Chem. Neue Folge, 1969, 65, 152.

6. Martín, M. T. and Möbius, D., Thin Solid Films, 1996, 284 $285,663$.

7. Sondermann, J., Liebigs Ann. Chem., 1971, 749, 183.

8. Fromherz, P., Rev. Sci. Instrum., 1975, 46, 1380.

9. Kuhn, H., Möbius, D. and Bücher, H., in Spectroscopy of Monolayer Assemblies, Vol. I, Part 3B. John Wiley and Sons, New York, 1972, p. 683.

10. Drexhage, K. H., Zwick, M. M. and Kuhn, H., Ber. Bunsenges. Phys. Chem., 1963, 67, 62. 